\title{
Automated Measures of Specific Vocabulary Knowledge from Constructed Responses ("Use These Words to Write a Sentence Based on this Picture")
}

\author{
Swapna Somasundaran \\ Educational Testing Services \\ 660 Rosedale Road, \\ Princeton, NJ 08541, USA \\ ssomasundaran@ets.org
}

\author{
Martin Chodorow \\ Hunter College and the Graduate Center \\ City University of New York, \\ New York, NY 10065, USA \\ martin. chodorowehunter. cuny. edu
}

\begin{abstract}
We describe a system for automatically scoring a vocabulary item type that asks test-takers to use two specific words in writing a sentence based on a picture. The system consists of a rule-based component and a machine learned statistical model which uses a variety of construct-relevant features. Specifically, in constructing the statistical model, we investigate if grammar, usage, and mechanics features developed for scoring essays can be applied to short answers, as in our task. We also explore new features reflecting the quality of the collocations in the response, as well as features measuring the consistency of the response to the picture. System accuracy in scoring is 15 percentage points greater than the majority class baseline and 10 percentage points less than human performance.
\end{abstract}

\section{Introduction}

It is often said that the best way to see if a person knows the meaning of a word is to have that person use the word in a sentence. Despite this widespread view, most vocabulary testing continues to rely on multiple choice items (e.g. (Lawless et al., 2012; Lawrence et al., 2012)). In fact, few assessments use constructed sentence responses to measure vocabulary knowledge, in part because of the considerable time and cost required to score such responses manually. While much progress has been made in automatically scoring writing quality in essays (Attali and Burstein, 2006; Leacock et al., 2014; Dale et al., 2012), the essay scoring engines do not measure proficiency in the use of specific words, except perhaps for some frequently confused homophones (e.g., its/it's, there/their/they're, affect/effect).
In this paper we present a system for automated scoring of targeted vocabulary knowledge based on short constructed responses in a picture description task. Specifically, we develop a system for scoring a vocabulary item type that is in operational use in English proficiency tests for nonnative speakers. Each task prompt in this item type consists of two target key words, for which the vocabulary proficiency is tested, and a picture that provides the context for the sentence construction. The task is to generate a single sentence, incorporating both key words, consistent with the picture. Presumably, a test-taker with competent knowledge of the key words will be able to use them in a well-formed grammatical sentence in the context of the picture.

Picture description tasks have been employed in a number of areas of study ranging from second language acquisition to Alzheimer's disease (Ellis, 2000; Forbes-McKay and Venneri, 2005). Pictures and picture-based story narration have also been used to study referring expressions (Lee et al., 2012) and to analyze child narratives in order to predict language impairment (Hassanali et al., 2013). Evanini et al. (2014) employ a series of pictures and elicit (oral) story narration to test English language proficiency. In our task, the picture is used as a constraining factor to limit the type and content of sentences that can be generated using the given key words.

In the course of developing our system, we examined existing features that have been developed for essay scoring, such as detectors of errors in grammar, usage and mechanics, as well as collocation features, to see if they can be re-used for scoring short responses. We also developed new features for assessing the quality of sentence construction using Pointwise Mutual Information (PMI). As our task requires responses to describe the prompt pictures, we manually constructed detailed textual descriptions of the pictures, and de- 
veloped features that measure the overlap between the content of the responses and the textual description. Our automated scoring system is partly based on deterministic scoring criteria and partly statistical. Overall, it achieves an accuracy of $76 \%$, which is a 15 percentage point improvement over a simple majority class baseline.

The organization of this paper is as follows: Section 2 describes the picture description task and the scoring guide that is used to manually score the picture description responses operationally. It also considers which aspects of scoring may be handled best by deterministic procedures and which are more amenable to statistical modeling. Section 3 details the construction of a reference corpus of text describing each picture, and Section 4 presents the features used in scoring. Section 5 describes our system architecture and presents our experiments and results. Detailed analysis is presented in Section 6, followed by related work in Section 7 and a summary with directions for future research in Section 8.

\section{Task Description and Data}

The picture description task is an item type that is in actual operational use as part of a test of English. It consists of a picture, along with two key words, one or both of which may be in an inflected form. Test-takers are required to use the two words in one sentence to describe the picture. They may change the inflections of the words as appropriate to the context of their sentence, but they must use some form of both words in one sentence. Requiring them to produce a response based on the picture constrains the variety of sentences and words that they are likely to generate.

Trained human scorers evaluate the responses based on appropriate use of grammar and the relevance of the sentence to the picture. The operational scoring guide is as follows:

score $=\mathbf{3}$ The response consists of ONE sentence that: (a) has no grammatical errors, (b) contains forms of both key words used appropriately, AND (c) is consistent with the picture.

score $=\mathbf{2}$ The response consists of one or more sentences that: (a) have one or more grammatical errors that do not obscure the meaning, (b) contain BOTH key words, (but they may not be in the same sentence and the form of the word(s) may not be accurate), AND (c) are consistent with the picture.

score $\mathbf{= 1}$ The response: (a) has errors that interfere with meaning, (b) omits one or both key words, OR (c) is not consistent with the picture.

score $=\mathbf{0}$ The response is blank, written in a foreign language, or consists of keystroke characters.

Our decisions about scoring system design are based on the scoring guide and its criteria. Some aspects of the scoring can be handled by simple pattern matching or lookup, while others require machine learning. For example, score 0 is assigned to responses that are blank or are not in English. This can be detected and scored in a straightforward way. On the other hand, the determination of grammaticality for the score points 3, 2 and 1 depends on the presence and severity of grammatical errors. A wide variety of such errors appear in responses, including errors of punctuation, subject-verb agreement, preposition usage and article usage. The severity of an error depends on how problematic the error is, and the system will have to learn this from the behavior of the trained human scorer(s), making this aspect of the scoring more amenable to statistical modeling.

Similarly, statistical modeling is more suitable for determining the consistency of the response with respect to the picture. According to the scoring guide, a response gets a score of 0 or 1 if it is not consistent with the picture, and gets a score of 2 or 3 if it is consistent. Thus, this aspect cannot solely determine the score of a response - it influences the score in conjunction with other language proficiency factors. Further, measures of how relevant a response is to a picture are likely to fall on a continuous scale, making a statistical modeling approach appropriate.

Finally, although there are some aspects of the scoring guide, such as the number of sentences and the presence of the key words, that can be measured trivially, they do not act as sole determinants of the score. For example, having more than one sentence can result in the response receiving a score of 2 or 1 . The number of sentences works in conjunction with other factors such as severity of grammar errors and relevance to the picture. Hence its contribution to the final score is best modeled statistically. 
As a result of the heterogeneous nature of the problem, our system is made up of a statistical learning component as well as a non-statistical component.

\subsection{Data}

The data set consists of about $58 \mathrm{~K}$ responses to 434 picture prompts. The mean response length was 11.26 words with a standard deviation of 5.10. The data was split into 2 development sets (consisting of a total of about $2 \mathrm{~K}$ responses) and a final train-test set (consisting of the remaining $56 \mathrm{~K}$ responses) used for evaluation. All $58 \mathrm{~K}$ responses were human scored using the scoring rubric discussed in Section 2. About 17K responses were double annotated. The inter-annotator agreement, using quadratic weighted kappa (QWK), was 0.83 . Score point 3 , the most frequent class, was assigned to $61 \%$ of the responses, followed by score point $2(31 \%)$, score point $1(7.6 \%)$ and score point $0(0.4 \%)$.

\section{Reference Picture Descriptions}

The pictures in our task vary in their complexity. A typical prompt picture might be a photograph of an outdoor marketplace, the inside of an airport terminal, a grocery store, a restaurant or a store room. Because consistency with respect to the picture is a crucial component in our task, we needed a reliable and exhaustive textual representation of each picture. Therefore, we manually constructed a reference text corpus for each of our 434 picture prompts. We chose to use manual creation of the reference corpus instead of trying automated image recognition because automated methods of image recognition are error prone and would result in a noisy reference corpus. Additionally, automated approaches would, at best, give us a (noisy) list of items that are present in the picture, but not the overall scene or event depicted.

Two annotators employed by a company that specializes in annotation created the reference corpora of picture descriptions. The protocol used for creating the reference corpus is shown below:

\section{Part-1: List the items, setting, and events in the picture.}

List, one by one, all the items and events you see in the picture. These may be animate objects (e.g. man), inanimate objects (e.g. table) or events (e.g. dinner). Try to capture both the overall setting (restaurant), as well as the objects that make up the picture (e.g. man, table, food). These are generally (but not necessarily) nouns and noun phrases. Some pictures can have many items, while some have only a few. The goal is to list 10-15 items and to capture as many items as possible, *starting with the most obvious ones*.

If the picture is too sparse, and you are not able to list at least 10 items, please indicate this as a comment.

\section{Part:2 Describe the picture}

Describe the scene unfolding in the picture. The scene in the picture may be greater than the sum of its parts (many of which you will list in part-1). For example, the objects in a picture could be "shoe" "man" "chair", but the scene in the picture could be that of a shoe purchase. The description tries to recreate the scene (or parts of the scene) depicted in the picture.

Generate a paragraph of 5-7 sentences describing the picture. Some of these sentences will address what is going on, while some may address relations between items. The proportions of these will differ, based on the picture. Make sure that you generate at least one sentence containing the two key words.

If the picture is too simple, and you are not able to generate at least 5 sentences, please indicate this as a comment.

The human annotator was given the picture and the two key words. The protocol for creating each reference corpus asked the annotator to first exhaustively list all the items (animate and inanimate) in the picture. Then, the annotator was asked to describe the scene in the picture. We used this two step process in order to capture, as much as possible, all objects, relationships between objects, settings and events depicted in the pictures.

The size of the reference corpus for each prompt is much larger than the single sentence test-taker response. This is intentional as the goal is to make the reference corpus as exhaustive as possible. We used a single annotator for each prompt. Double annotation using a secondary annotator was done in cases where we felt that the coverage of the corpus created by the primary annotator was insuffi- 
cient ${ }^{1}$.

In order to test coverage, we used a small development set of essays from each prompt and compared the coverage of the generated reference corpus over the development essays. If the coverage (proportion of content words in the responses that were found in the reference corpus) was less than $50 \%$ (this was the case for about $20 \%$ of the prompts), we asked the secondary annotator to create a new reference corpus for the prompt. The two reference corpora for the prompt were then simply combined to form a single reference corpus.

\section{Features for automated scoring}

Because the score points in the scoring guide conflate, to some degree, syntactic, semantic, and other weaknesses in the response, we carried out a scoring study on a second small development set (comprising of a total of 80 responses from 4 prompts, picked randomly) to gather insight into the general problems in English language proficiency exhibited in the responses. For the study, it was necessary to have test-taker responses rescored by an annotator using an analytic scheme which makes the types and locations of problems explicit. This exercise revealed that, in addition to the factors stated explicitly in the scoring guide, there is another factor that results in low comprehension (readability) of the sentence and that reflects lower English proficiency. Specifically, the annotator tagged many sentences as being "awkward". This awkwardness was due to poor choice of words or to poor construction of the sentence. For example, in the sentence "The man is putting some food in bags while he is recording for the payment", "recording for the payment" was marked as an awkward phrase. Based on our annotation of the scores and on the descriptions in the scoring guide, we selected features designed to capture grammar, picture relevance and awkward usage. We discuss each of our feature sets in the following subsections.

\subsection{Features for Grammatical Error Detection}

Essay scoring engines such as e-rater ${ }^{\circledR}$ (Attali and Burstein, 2006) typically use a number of

\footnotetext{
${ }^{1}$ We do not conduct inter-annotator agreement studies as the goal of the double annotation was to create a diverse description.
}

grammar, usage and mechanics features that detect and quantify different types of English usage errors in essays. Examples of some of these error types are: Run-on Sentences, Subject Verb Agreement Errors, Pronoun Errors, Missing Possessive Errors, Wrong Article Errors, Missing Article Errors, Preposition Errors, Non-standard Verb or Word Form Errors, Double Negative Errors, Fragment or Missing Comma Errors, Ill-formed Verb Errors, Wrong Form of Word Errors, Spelling Errors, Wrong Part of Speech Errors, and Missing Punctuation Errors .

In addition to these, essay scoring engines often also use as features the Number of Sentences that are Short, the Number of Sentences that are Long, the Number of Passive Sentences, and other features that are relevant only for longer texts such as essays. Accordingly, we selected, from e-rater 113 grammar, word usage, mechanics and lexical complexity features that could be applied to our short response task. This forms our grammar feature set.

\subsection{Features for Measuring Content Relevance}

We generated a set of features that measure the content overlap between a given response and the corresponding reference corpus for the prompt. For this, first the keywords and the stop words were removed from the response and the reference corpus, and then the proportion of overlap was calculated between the lemmatized content words of the response and the lemmatized version of the corresponding reference corpus, as follows:

$$
\frac{\mid \text { Response } \cap \text { Corpus } \mid}{\mid \text { Response } \mid}
$$

It is not always necessary for the test-taker to use exactly the same words found in the reference corpus. For example, the annotator might have referred to a person in the picture as a "lady", while a response may refer to the same person as a "woman" or "girl" or even just "person". Thus, we needed to go beyond simple lexical match. In order to account for synonyms, we expanded the content words in the reference corpus by adding their synonyms, as provided in Lin's thesaurus (Lin, 1998) and then compared the expanded reference to each response. Along the same lines, we also used expansions from WordNet synonyms, WordNet hypernyms and WordNet hyponyms. The following is the list of our content 
relevance features. Each measures the proportion of overlap as described by the equation above between the lemmatized response and

1. lemmas: the lemmatized reference corpus.

2. cov-lin: the reference corpus expanded using Lin's thesaurus.

3. cov-wn-syns: the reference corpus expanded using WordNet Synonyms.

4. cov-wn-hyper: the reference corpus expanded using WordNet Hypernyms.

5. cov-wn-hypo: the reference corpus expanded using WordNet Hyponyms.

6. cov-all: the reference corpus expanded using all of the above methods.

Mean proportions of overlap ranged from 0.65 for lemmas to 0.97 for cov-all.

The 6 features listed above, along with the prompt id give a total of 7 features that form our relevance feature set. We use prompt id as a feature because the extent of overlap can depend on the prompt. Some pictures are very sparse, so, the description of the picture in the response will be short, and will not vary much from the reference corpus. For these, a good amount of overlap between the response and reference corpus is expected. Other pictures are very dense with a large number of objects and items shown. In this case, any single response may describe just a small subset of the items and satisfy the consistency criteria, and consequently, even a small overlap between the response and the reference corpus may be sufficient.

\subsection{Features for Awkward Word Usage}

In order to measure awkward word usage, we explored PMI-based features, and also investigated whether some features developed for essay scoring can be used effectively for this purpose.

\subsubsection{PMI-based ngram features}

Non-native writing is often characterized by inappropriate combinations of words, indicating the writer's lack of knowledge of collocations. For example, "recording for the payment" might be better expressed as "entering the price in the cash register". As "recording for the payment" is an inappropriate construction, it is not likely to be common, for example, in a large web corpus. We use this intuition in constructing our PMI-based features.

We find the PMI of all adjacent word pairs (bigrams), as well as all adjacent word triples (trigrams) in the Google 1T web corpus (Brants and Franz, 2006) using the TrendStream database (Flor, 2013).

PMI between word pairs (bigram $\mathrm{AB}$ ) is defined as:

$$
\log _{2} \frac{p(A B)}{p(A) \cdot p(B)}
$$

and between word triples (trigram $\mathrm{ABC}$ ) as

$$
\log _{2} \frac{p(A B C)}{p(A) \cdot p(B) \cdot p(C)}
$$

The higher the value of the PMI, the more common is the collocation for the word pair/triple in well formed texts. On the other hand, negative values of PMI indicate that the given word pair (or triple) is less likely than chance to occur together. We hypothesized that this would be a good indicator of awkward usage, as suggested in (Chodorow and Leacock, 2000).

The PMI values for adjacent words obtained over the entire response are then assigned to bins, with 8 bins for word pairs and another 8 for word triples. Each bin represents a range for PMI $p$ taking real values $\mathbb{R}$ as follows:

$$
\begin{gathered}
\text { bin }_{1}=\{p \in \mathbb{R} \mid p>20\} \\
\text { bin }_{2}=\{p \in \mathbb{R} \mid 10<p \leq 20\} \\
\text { bin }_{3}=\{p \in \mathbb{R} \mid 1<p \leq 10\} \\
\text { bin }_{4}=\{p \in \mathbb{R} \mid 0<p \leq 1\} \\
\text { bin }_{5}=\{p \in \mathbb{R} \mid-1<p \leq 0\} \\
\text { bin }_{6}=\{p \in \mathbb{R} \mid-10<p \leq-1\} \\
\text { bin }_{7}=\{p \in \mathbb{R} \mid-20<p \leq-10\} \\
\text { bin }_{8}=\{p \in \mathbb{R} \mid p \leq-20\}
\end{gathered}
$$

Once the PMI values for the adjacent word pairs in the response are generated, we generate two sets of features. The first set is based on the counts of word pairs falling into each bin (for example, Number of pairs falling into bin 1 , Number of pairs falling into bin 2 and so on). The second set of features are based on percentages (for example Percentage of pairs falling into bin 1 , Percentage of pairs falling into bin ${ }_{2}$ etc.). These two sets result in a total of 16 features. We similarly generate 16 more features for adjacent word triples. We 
use percentages in addition to raw counts to account for the length of the response. For example, it is possible for a long sentence to have phrases that are awkward as well as well formed, giving the same counts of phrases in the high-PMI value bins as that of a short sentence that is entirely well formed.

In addition to binning, we also encode as features the maximum, minimum and median PMI value obtained over all word pairs. The first two features capture the best and the worst word collocations in a response. The median PMI value captures the overall general quality of the response in a single number. For example, if this is a low number, then the response generally has many bad phrasal collocations. Finally a null-PMI feature is used to count the number of pairs that had zero entries in the database. This feature is an indicator that the given words or word collocations were not found even once in the database. Given the size of the underlying database, this usually happens in cases when words are misspelled, or when the words never occur together.

All features created for bigrams are also created for trigrams. We thus have a total of 40 features, called the $p m i$ feature set.

\subsubsection{Features from essay scoring}

A number of measures of collocation quality have been proposed and implemented (e.g. (Futagi et al., 2008; Dahlmeier and Ng, 2011)). We use erater's measure of the density of 'good' collocations found in the response. Another source of difficulty for non-native writers is the selection of appropriate prepositions. We use the mean probability assigned by e-rater to the prepositions in the response. These two measures, one for the quality of collocations and the other for the quality of prepositions, are combined in our colprep feature set.

\subsection{Scoring Rubric-based Features}

As seen in Section 2, some of the criteria for scoring are quite straightforward (e.g. "omits one or both key words"). While these are not sole determinants of a score, they are certainly strong influences. Thus, we encode four criteria from the scoring guide. These form our final feature set, rubric, and are binary values, answering the questions: Is the first key word from the prompt present in the response? Is the second key word from the prompt present in the response? Are both key words from the prompt present in the response? Is there more than one sentence in the response?

Table 1 provides a list of feature types and the corresponding number of features of each type.

\begin{tabular}{|c|c|}
\hline Feature set type & Number of Features \\
\hline grammar & 113 \\
\hline relevance & 7 \\
\hline pmi & 40 \\
\hline colprep & 2 \\
\hline rubric & 4 \\
\hline
\end{tabular}

Table 1: Feature sets and the counts of features in each set

\section{System and Evaluation}

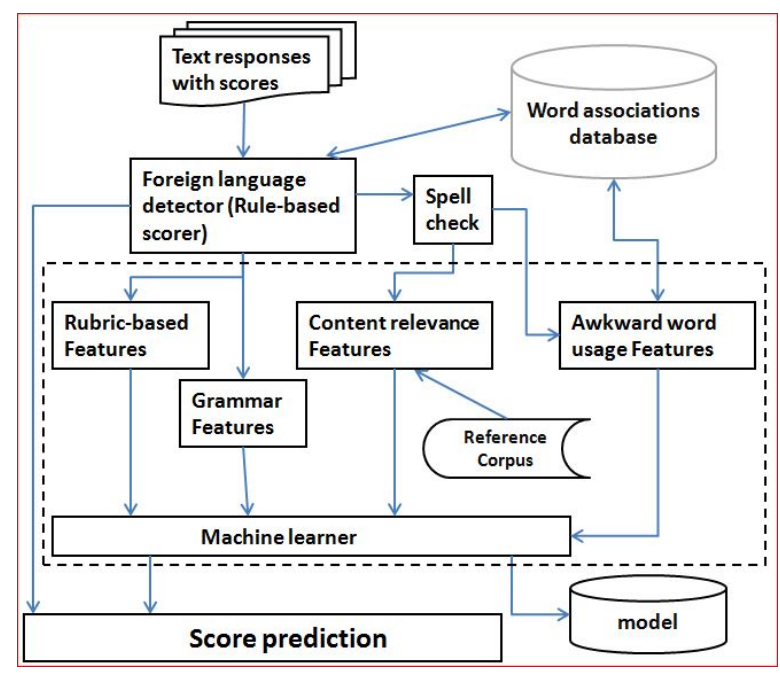

Figure 1: System Architecture

As noted earlier, the system is partly rule-based and partly statistical. Figure 1 illustrates the system architecture. The rule-based part captures the straightforward deterministic scoring criteria while the machine learning component encodes features described in Section 4 and learns how to weight the features for scoring based on humanscored responses.

As described in Section 2, detection of conditions that result in a score of zero are straightforward. Our rule-based scorer (shown as "Foreign Language Detector" in Figure 1) assigns a zero score to a response if it is blank or nonEnglish. The system determines if the response is non-English based on the average of PMI bigram scores over the response. If the average score is less than a threshold value, the system tags it as 
a non-English sentence. The threshold was determined by manually inspecting the PMI values obtained for sentences belonging to English and nonEnglish news texts. Responses given zero scores by this module are filtered out and do not go to the next stage.

Responses that pass the rule-based scorer are then sent to the statistical scorer. Here, we encode the features discussed in Section 4. Spell checking and correction are carried out before features for content relevance and PMI-based awkward word usage are computed. This is done in order to prevent misspellings from affecting the reference corpus match or database search. The original text is sent to the Grammar feature generator as it creates features based on misspellings and other word form errors. Finally, we use all the features to train a Logistic Regression model using sklearn. Note that the statistical system predicts all 4 scores $(0$ through 3). This is because the rule-based system is not perfect; that is, it might miss some responses that should receive zero scores, and pass them over to the next stage.

\subsection{Metrics}

We report our results using overall accuracy, quadratic weighted kappa (QWK) and score-level precision, recall and f-measure. The precision $P$ of the system is calculated for each score point $i$ as

$$
P_{i}=\frac{\left|S_{i} \cap H_{i}\right|}{\left|S_{i}\right|}
$$

where $\left|S_{i}\right|$ is the number of responses given a score of $i$ by the system, and $\left|S_{i} \cap H_{i}\right|$ is the number of responses given a score of $i$ by the system as well as the human rater.

Similarly, recall, $R$ is calculated for each score point $i$ as

$$
R_{i}=\frac{\left|S_{i} \cap H_{i}\right|}{\left|H_{i}\right|}
$$

F-measure $F_{i}$ is calculated as the harmonic mean of the precision $P_{i}$ and recall $R_{i}$ at each score point $i$. Accuracy is the ratio of the number of responses correctly classified over the total number of responses.

\subsection{Results}

All of the responses in the train-test set were passed through the rule-based zero-scorer. A total of 210 responses had been scored as zero by the human scorer. The rule-based system scored 222 responses as zeros, of which 184 were correct.
The precision $P^{\text {rule }}$ of the rule-based system is calculated as

$$
P_{0}^{\text {rule }}=\frac{184}{222}=82.9 \%
$$

Similarly, Recall is calculated as

$$
R_{0}^{\text {rule }}=\frac{184}{210}=87.6 \%
$$

The corresponding F-measure is $85.2 \%$

The remaining responses pass to the next stage where machine learning is employed. We performed 10 fold cross-validation experiments using Logistic Regression as well as Random Forest learners. As the results are comparable, we only report those from logistic regression.

\begin{tabular}{|c|c|c|}
\hline & Accuracy in \% & Agreement (QWK) \\
\hline \hline Baseline & 61.00 & - \\
\hline System & 76.23 & 0.63 \\
\hline Human & 86.00 & 0.83 \\
\hline
\end{tabular}

Table 2: Overall system and human accuracy (in percentage) and agreement (using Quadratic Weighted Kappa)

Table 2 reports the results. The system achieves an accuracy of $76.23 \%$, which is more than a 15 percentage point improvement over the majority class baseline of $61 \%$. The majority class baseline always predicts a score of 3 . Compared to human performance, system performance is 10 percentage points lower (human-human agreement is $86 \%$ ). Quadratic weighted kappa for systemhuman agreement is also lower (0.63) than for human-human agreement (0.83).

Table 3 reports the precision, recall and $\mathrm{F}$ measure of the system for each of the score points.

\begin{tabular}{|c|c|c|c|}
\hline Score point & Precision & Recall & F-measure \\
\hline \hline 0 & 84.2 & 68.3 & 72.9 \\
\hline 1 & 78.4 & 67.5 & 72.6 \\
\hline 2 & 70.6 & 50.4 & 58.8 \\
\hline 3 & 77.8 & 90.5 & 83.6 \\
\hline
\end{tabular}

Table 3: Overall system performance at each score point using all features

\section{Analysis}

In order to understand the usefulness of each feature set in scoring the responses, we constructed 
systems using first the individual features alone, and then using feature combinations. Table 4 reports the accuracy of the learner using individual features alone. We see that, individually, each feature set performs much below the performance of the full system (that has an accuracy of $76.23 \%$ ), which is expected, as each feature set represents a particular aspect of the construct. However, in general, each of the feature-sets (except colprep) shows improvement over baseline, indicating that they contribute towards performance improvement in the automated system.

Grammar features are the best of the individual feature sets at $70 \%$ accuracy, indicating that grammatical error features developed for longer texts can be applied to single sentences. The PMIbased feature set is the second best performer, indicating its effectiveness in capturing word usage issues. While colprep and pmi both capture awkward usage, pmi alone shows better performance $(67.44 \%)$ than colprep alone $(61.26 \%)$. Also, when rubric is used alone, the resulting system produces a four percentage point improvement over the baseline, with $65 \%$ accuracy, indicating the presence of responses where the test-takers are not able to incorporate one or both words in a single sentence. The relevance feature set by itself does not show substantial improvement over the baseline. This is not surprising, as according to the scoring guide, a response gets a score of 0 or 1 if it does not describe the picture, and gets a score of 2 or 3 if it is relevant to the picture. Hence, this feature cannot solely and accurately determine the score.

\begin{tabular}{|c|c|}
\hline Feature Set & Accuracy in \% \\
\hline \hline grammar & 70.30 \\
\hline pmi & 67.44 \\
\hline rubric & 65.00 \\
\hline relevance & 62.50 \\
\hline colprep & 61.26 \\
\hline
\end{tabular}

Table 4: System performance for individual features

Table 5 reports accuracies of systems built using feature set combinations. The first feature set combination, grammar + colprep, is a set of all features obtained from essay scoring. Here we see that addition of colprep does not improve the performance over that obtained by grammar features alone. Further, when colprep is combined with pmi (colprep+pmi, row 2), there is a slight drop in performance as compared to using pmi-based features alone. These results indicate that colprep, while being useful for larger texts, does not transfer well to the simple single sentence responses in our task.

Further, in Table 5 we see that the system using a combination of the pmi feature set and the relevance feature set (pmi+relevance) achieves an accuracy of $69 \%$. Thus, this feature combination is able to improve performance over that using either feature set alone, indicating that while content relevance features by themselves do not create an impact, they can improve performance when added to other features. Finally, the feature combination of all new features developed for this task ( $p m i+$ relevance + rubric) yields $73 \%$ accuracy, which is again better than each individual feature set's performance, indicating that they can be synergistically combined to improve system performance.

\begin{tabular}{|c|c|}
\hline Feature Set & Accuracy in \% \\
\hline \hline (i) grammar + colprep & 70.31 \\
\hline (ii) colprep + pmi & 67.42 \\
\hline (iii) pmi + relevance & 69.05 \\
\hline (iv) pmi + relevance + rubric & 73.21 \\
\hline
\end{tabular}

Table 5: System performance for feature combinations (i) typically used in essay scoring, (ii) that measure awkwardness, (iii) newly proposed here, (iv) newly proposed plus rubric-specific criteria

\section{Related Work}

Most work in automated scoring and learner language analysis has focused on detecting grammar and usage errors (Leacock et al., 2014; Dale et al., 2012; Dale and Narroway, 2012; Gamon, 2010; Chodorow et al., 2007; Lu, 2010). This is done either by means of handcrafted rules or with statistical classifiers using a variety of information. In the case of the latter, the emphasis has been on representing the contexts of function words, such as articles and prepositions. This work is relevant inasmuch as errors in using content words, such as nouns and verbs, are often reflected in the functional elements which accompany them, for example, articles that indicate the definiteness or countability of nouns, and prepositions that mark the cases of the arguments of verbs.

Previous work (Bergsma et al., 2009; Bergsma et al., 2010; Xu et al., 2011) has shown that mod- 
els which rely on large web-scale n-gram counts can be effective for the task of context-sensitive spelling correction. Measures of ngram association such as PMI, log likelihood, chi-square, and $t$ have a long history of use for detecting collocations and measuring their quality (see (Manning and Schütze, 1999) and (Leacock et al., 2014) for reviews). Our application of a large n-gram database and PMI is to detect inappropriate word usage.

Our task also differs from work focusing on evaluating content (e.g. (Meurers et al., 2011; Sukkarieh and Blackmore, 2009; Leacock and Chodorow, 2003)) in that, although we are looking for usage of certain content words, we focus primarily on measuring knowledge of vocabulary.

Recent work on assessment measures of depth of vocabulary knowledge (Lawless et al., 2012; Lawrence et al., 2012), has argued that knowledge of specific words can range from superficial (idiomatic associations built up through word cooccurrence) to topical (meaning-related associations between words) to deep (definitional knowledge). Some of our features (e.g. awkward word usage) capture some of this information (e.g., idiomatic associations between words), but assigning the depth of knowledge of the key words is not the focus of our task.

Work that is closely related to ours is that of King and Dickinson (2013). They parse picture descriptions from interactive learner sentences, classify sentences into syntactic types and extract the logical subject, verb and object in order to recover simple semantic representations of the descriptions. We do not explicitly model the semantic representations of the pictures, but rather our goal in this work is to ascertain if a response is relevant to the picture and to measure other factors that reflect vocabulary proficiency.

We employ human annotators and use word similarity measures to obtain alternative forms of description because the proprietary nature of our data prevents us from releasing our pictures to the public. However, crowd sourcing has been used by other researchers to collect human labels for images and videos. For example, Rashtchian et al. (2010) use Amazon Mechanical Turk and Von Ahn and Dabbish (2004) create games to entice players to correctly label images. Chen and Dolan (2011) use crowd sourcing to collect multiple paraphrased descriptions of videos to create a paraphrasing corpus.

In a vast body of related work, automated methods have been explored for the generation of descriptions of images (Kulkarni et al., 2013; Kuznetsova et al., 2012; Li et al., 2011; Yao et al., 2010; Feng and Lapata, 2010a; Feng and Lapata, 2010b; Leong et al., 2010; Mitchell et al., 2012). There is also work in the opposite direction, of finding or generating pictures for a given narration. Joshi et al. (2006) found the best set of images from an image database to match the keywords in a story. Coyne and Sproat (2001) developed a natural language understanding system which converts English text into threedimensional scenes that represent the text. For a high-stakes assessment, it would be highly undesirable to have any noise in the gold-standard reference picture descriptions. Hence we chose to use manual description for creating our reference corpus.

\section{Summary and Future Directions}

We investigated different types of features for automatically scoring a vocabulary item type which requires the test-taker to use two words in writing a sentence based on a picture. We generated a corpus of picture descriptions for measuring the relevance of responses, and as a foundation for feature development, we performed preliminary fine-grained annotations of responses. The features used in the resulting automated scoring system include newly developed statistical measures of word usage and response relevance, as well as features that are currently found in essay scoring engines. System performance shows an overall accuracy in scoring that is 15 percentage points above the majority class baseline and 10 percentage points below human performance.

There are a number of avenues open for future exploration. The automated scoring system might be improved by extending the relevance feature to include overlap with previously collected highscoring responses. The reference corpus could also be expanded and diversified by using a large number of annotators, at least some of whom are speakers of the languages that are most prominently represented in the population of test-takers. Finally, one particular avenue we would like to explore is the use of our features to provide feedback in low stakes practice environments. 


\section{References}

Yigal Attali and Jill Burstein. 2006. Automated essay scoring with e-rater v. 2.0. Journal of Technology, Learning, and Assessment, 4:3.

Shane Bergsma, Dekang Lin, and Randy Goebel. 2009. Web-scale n-gram models for lexical disambiguation. In IJCAI.

Shane Bergsma, Emily Pitler, and Dekang Lin. 2010. Creating robust supervised classifiers via web-scale n-gram data. In Proceedings of the 48th Annual Meeting of the Association for Computational Linguistics, pages 865-874. Association for Computational Linguistics.

Thorsten Brants and Alex Franz. 2006. Web 1T 5gram Version 1. In Linguistic Data Consortium, Philadelphia.

David L Chen and William B Dolan. 2011. Collecting highly parallel data for paraphrase evaluation. In Proceedings of the 49th Annual Meeting of the Association for Computational Linguistics: Human Language Technologies-Volume 1, pages 190-200. Association for Computational Linguistics.

Martin Chodorow and Claudia Leacock. 2000. An unsupervised method for detecting grammatical errors. In Proceedings of the Conference of the North American Chapter of the Association of Computational Linguistics (NAACL), pages 140-147.

Martin Chodorow, Joel R Tetreault, and Na-Rae Han. 2007. Detection of grammatical errors involving prepositions. In Proceedings of the fourth ACLSIGSEM workshop on prepositions, pages 25-30. Association for Computational Linguistics.

Bob Coyne and Richard Sproat. 2001. Wordseye: an automatic text-to-scene conversion system. In Proceedings of the 28th annual conference on Computer graphics and interactive techniques, pages 487-496. ACM.

Daniel Dahlmeier and Hwee Tou Ng. 2011. Correcting semantic collocation errors with $\mathrm{L} 1$ induced paraphrases. In Proceedings of the Conference on Empirical Methods in Natural Language Processing, EMNLP '11, pages 107-117, Stroudsburg, PA, USA. Association for Computational Linguistics.

Robert Dale and George Narroway. 2012. A framework for evaluating text correction. In $L R E C$, pages 3015-3018.

Robert Dale, Ilya Anisimoff, and George Narroway. 2012. HOO 2012: A report on the preposition and determiner error correction shared task. In Proceedings of the Seventh Workshop on Building Educational Applications Using NLP, pages 54-62. Association for Computational Linguistics.

Rod Ellis. 2000. Task-based research and language pedagogy. Language teaching research, 4(3):193220
Keelan Evanini, Michael Heilman, Xinhao Wang, and Daniel Blanchard. 2014. Automated scoring for TOEFL Junior comprehensive writing and speaking. Technical report, ETS, Princeton, NJ.

Yansong Feng and Mirella Lapata. 2010a. How many words is a picture worth? Automatic caption generation for news images. In Proceedings of the 48th Annual Meeting of the Association for Computational Linguistics, ACL'10, pages 1239-1249, Stroudsburg, PA, USA. Association for Computational Linguistics.

Yansong Feng and Mirella Lapata. 2010b. Topic models for image annotation and text illustration. In Human Language Technologies: The 2010 Annual Conference of the North American Chapter of the Association for Computational Linguistics, HLT '10, pages 831-839, Stroudsburg, PA, USA. Association for Computational Linguistics.

Michael Flor. 2013. A fast and flexible architecture for very large word n-gram datasets. Natural Language Engineering, 19(1):61-93.

KE Forbes-McKay and Annalena Venneri. 2005. Detecting subtle spontaneous language decline in early Alzheimers disease with a picture description task. Neurological sciences, 26(4):243-254.

Yoko Futagi, Paul Deane, Martin Chodorow, and Joel Tetreault. 2008. A computational approach to detecting collocation errors in the writing of non-native speakers of English. Computer Assisted Language Learning, 21(4):353-367.

Michael Gamon. 2010. Using mostly native data to correct errors in learners' writing: A meta-classifier approach. In Human Language Technologies: The 2010 Annual Conference of the North American Chapter of the Association for Computational Linguistics, pages 163-171. Association for Computational Linguistics.

Khairun-nisa Hassanali, Yang Liu, and Thamar Solorio. 2013. Using Latent Dirichlet Allocation for child narrative analysis. ACL 2013, page 111.

Dhiraj Joshi, James Z. Wang, and Jia Li. 2006. The story picturing engine-a system for automatic text illustration. ACM Trans. Multimedia Comput. Commun. Appl., 2(1):68-89, February.

Levi King and Markus Dickinson. 2013. Shallow semantic analysis of interactive learner sentences. In Proceedings of the Eighth Workshop on Innovative Use of NLP for Building Educational Applications, pages 11-21, Atlanta, Georgia, June. Association for Computational Linguistics.

Girish Kulkarni, Visruth Premraj, Vicente Ordonez, Sagnik Dhar, Siming Li, Yejin Choi, Alexander C. Berg, and Tamara L. Berg. 2013. Babytalk: Understanding and generating simple image descriptions. IEEE Transactions on Pattern Analysis and Machine Intelligence, 99(PrePrints):1. 
Polina Kuznetsova, Vicente Ordonez, Alexander C Berg, Tamara L Berg, and Yejin Choi. 2012. Collective generation of natural image descriptions. In Proceedings of the 50th Annual Meeting of the Association for Computational Linguistics: Long Papers-Volume 1, pages 359-368. Association for Computational Linguistics.

René Lawless, John Sabatini, and Paul Deane. 2012. Approaches to assessing partial vocabulary knowledge and supporting word learning: Assessing vocabulary depth. In Annual Meeting of the American Educational Research Association, April 13-17, 2012, Vancouver, $C A$.

Joshua Lawrence, Elizabeth Pare-Blagoev, René Lawless, and Chen Deane, Paul andLi. 2012. General vocabulary, academic vocabulary, and vocabulary depth: Examiningpredictors of adolescent reading comprehension. In Annual Meeting of the American Educational Research Association.

Claudia Leacock and Martin Chodorow. 2003. C-rater: Automated scoring of short-answer questions. Computers and the Humanities, 37(4):389-405.

Claudia Leacock, Martin Chodorow, Michael Gamon, and Joel Tetreault. 2014. Automated Grammatical Error Detection for Language Learners. Synthesis Lectures on Human Language Technologies. Morgan \& Claypool.

Choonkyu Lee, Smaranda Muresan, and Karin Stromswold. 2012. Computational analysis of referring expressions in narratives of picture books. NAACL-HLT 2012, page 1.

Chee Wee Leong, Rada Mihalcea, and Samer Hassan. 2010. Text mining for automatic image tagging. In Proceedings of the 23rd International Conference on Computational Linguistics: Posters, pages 647-655. Association for Computational Linguistics.

Siming Li, Girish Kulkarni, Tamara L Berg, Alexander C Berg, and Yejin Choi. 2011. Composing simple image descriptions using web-scale n-grams. In Proceedings of the Fifteenth Conference on Computational Natural Language Learning, pages 220 228. Association for Computational Linguistics.

Dekang Lin. 1998. Automatic retrieval and clustering of similar words. In Proceedings of the 17th international conference on Computational linguisticsVolume 2, pages 768-774. Association for Computational Linguistics.

Xiaofei Lu. 2010. Automatic analysis of syntactic complexity in second language writing. International Journal of Corpus Linguistics, 15(4).

Christopher D. Manning and Hinrich Schütze. 1999. Foundations of Statistical Natural Language Processing. MIT Press.
Detmar Meurers, Ramon Ziai, Niels Ott, and Stacey M Bailey. 2011. Integrating parallel analysis modules to evaluate the meaning of answers to reading comprehension questions. International Journal of Continuing Engineering Education and Life Long Learning, 21(4):355-369.

Margaret Mitchell, Xufeng Han, Jesse Dodge, Alyssa Mensch, Amit Goyal, Alex Berg, Kota Yamaguchi, Tamara Berg, Karl Stratos, and Hal Daumé III. 2012. Midge: Generating image descriptions from computer vision detections. In Proceedings of the 13th Conference of the European Chapter of the Association for Computational Linguistics, pages 747756. Association for Computational Linguistics.

Cyrus Rashtchian, Peter Young, Micah Hodosh, and Julia Hockenmaier. 2010. Collecting image annotations using Amazon's Mechanical Turk. In Proceedings of the NAACL HLT 2010 Workshop on Creating Speech and Language Data with Amazon's Mechanical Turk, pages 139-147. Association for Computational Linguistics.

Jana Zuheir Sukkarieh and John Blackmore. 2009. C-rater: Automatic content scoring for short constructed responses. In FLAIRS Conference.

Luis Von Ahn and Laura Dabbish. 2004. Labeling images with a computer game. In Proceedings of the SIGCHI conference on Human factors in computing systems, pages 319-326. ACM.

Wei Xu, Joel Tetreault, Martin Chodorow, Ralph Grishman, and Le Zhao. 2011. Exploiting syntactic and distributional information for spelling correction with web-scale n-gram models. In Proceedings of the Conference on Empirical Methods in Natural Language Processing, pages 1291-1300. Association for Computational Linguistics.

Benjamin Z Yao, Xiong Yang, Liang Lin, Mun Wai Lee, and Song-Chun Zhu. 2010. I2t: Image parsing to text description. Proceedings of the IEEE, 98(8):1485-1508. 